and epinephrine used together versus epinephine alone in out-ofhospital cardiac arrest (CA).

Methods We searched MEDLINE for randomised trials comparing the efficacy of vasopressin and epinephrine versus epinephrine alone in adults who experienced out-of-hospital CA. The primary outcome was the return of spontaneous circulation (ROSC) and the survival rate on admission and discharge. We also analysed ROSC in subgroups of patients presenting with different arrest rhythms.

Results In total, 206 articles were enrolled and five studies were included. No differences were found between these groups (vasopressin and epinephrine group vs. epinephrine alone group), except for the survival rate at $24 \mathrm{~h}$ (OR 2.99, 95\% CI 1.43, 6.28). No evidence supports the conclusion that vasopressin combined with epinephrine is better than epinephrine alone for ROSC, even amongst subgroups of patients.

Conclusion This systematic review of the efficacy of vasopressin and epinephrine found that its combined use is better for $24 \mathrm{~h}$ survival rate but only in one study which included 122 patients. Further investigation will be needed to support the -use of this combination for out-of-hospital CA management.

\section{e0667 THE ROLE OF RANTES FACTOR IN THE STUDY OF HYPERGLYCEMIA AND CORONARY HEART DISEASE}

doi:10.1136/hrt.2010.208967.667

Yang Lixia, Qi Feng, Guo Ruiwei, Wang Xianmei, Guo Chuanming. Department of Cardiology Kunmin General Hospital of Chinese Plakunming

Objective To study the correlative relationship of activated $\mathrm{T}$ cell chemokine (RANTES) and high blood glucose and coronary heart disease, and investigate the role of RANTES factor in hyperglycemia and coronary heart disease.

Methods The 360 patients were divided into Coronary Heart Disease $(\mathrm{CHD})$ group $(n=300)$ and control group $(n=60)$ according to the Coronary Angiography (CAG), and CHD group were divided into acute coronary syndrome (ACS) group $(n=180)$ and stable angina pectoris (SAP) group $(n=120)$. The severity and extent of coronary lesions was analysed by CAG and typified by means of Gensini coronary score system. Blood samples of ACS patients were taken immediately on admission, and the $12 \mathrm{~h}$ fasting blood samples of other patients were taken in the day after admission, all patients were taken $3 \mathrm{ml}$ blood from elbow vein and put into anticoagulant tube. Then all samples were centrifuged for $10 \mathrm{~min}$ with the speed of $3000 \mathrm{r} / \mathrm{min}$, and the separated serum was frozen at $-80^{\circ} \mathrm{C}$ refrigerator waiting for test. Linked immunosorent assay was used to measure the RANTES concentration. We expressed the level of RANTES and other biochemical indicators in all groups with, and compare the differences between the three groups using AVONA (analysis of variance). And then, $\mathrm{q}$ test was used for pairwise comparison; multiple regression equation was used for analysing the relationships of RANTES chemokine, blood glucose and coronary artery disease; Spearman's correlation coefficient was used for analysing the correlation of RANTES chemokine and blood glucose. Results Significantly increasing of RANTES concentration was observed in ACS group $(222.57 \pm 28.55 \mathrm{pg} / \mathrm{ml}$ ) compared to the SAP group $(199.77 \pm 22.20 \mathrm{pg} / \mathrm{ml}$ ) and the control group (162.06 $\pm 13.15 \mathrm{pg} /$ $\mathrm{ml})(\mathrm{p}<0.05)$. Positive correlation were seen between RANTE chemokines, fasting glucose, LDL-C and the Gensini score of coronary artery lesions $(\mathrm{p}<0.05)$. Hyperglycemia was positively correlated with RANTES concentration $(\mathrm{r}=0.69, \mathrm{p}<0.05)$.

Conclusions Hyperglycemia plays an important role in occurrence and prognosis of acute coronary syndrome (ACS), and blood glucose was positively correlated with RANTES concentration. The increasing of RANTES levels consistent with the increasing of the risk of type 2 diabetes, it plays an important role in various complications of diabetes, and blood sugar, RANTES associated with coronary artery disease. RANTES factor may play an important role in high blood sugar and coronary artery inflammation complications, especially for the stability of ACS vulnerable plaque. The mechanism is that high glucose and its metabolites end products (AGE) may be play an important role in the incidence of coronary heart disease, especially in ACS by the NF-K $\beta /$ RANTES/ MMP-9 ways.

\section{e0668 STUDY ON THE ANTI-OXIDATIVE FUNCTION OF KOREAN MONKSHOOD ROOT POLYSACCHARIDE}

doi:10.1136/hrt.2010.208967.668

Sun Xin, Gong Zhanwei, Gao Lin, Wang Qi, Gao Xin, Lv Gang. Life Science Center, Beihua University, China

Objective Exploring the anti-oxidative function of traditional Chinese herbal-Korean Monkshood Root polysaccharide.

Methods Using GE Corporation's KTA explore FPLC purification system, we separate and purify the Korean Monkshood Root polysaccharide. After establishing the anti-oxidation experimental model, experimental study of elimination of the ultra oxygen anion and DPPH free radical are carried out.

Results As the active ingredient, Korean Monkshood Root polysaccharides can remove $\mathrm{O}^{2-}$ and DPPH free radical, which shows that Korean Monkshood Root polysaccharide can eliminate free radical and has the function of anti-oxidation.

Conclusions Korean Monkshood Root polysaccharides can be used as a natural anti-oxidation for human cardiovascular disease treatment and preventive health care.

\section{Related Subjects: Kidney and Cardiovascular Disease \\ e0669 ERYTHROPOIETIN COMBINED WITH L-CARNITINE TREATMENT OF RENAL ANAEMIA ON ENDOTHELIN AND LEFT VENTRICULAR REMODELLING}

doi:10.1136/hrt.2010.208967.669

Hui Li, Shu Sun, Yumei Wu. Affliated First People's Hospital of Jining of Shandong Academy of Medical Sciences

Objective To observe the effects of Xuezhikang on blood lipids and the levels of plasma endothelins, thromboxane $\mathrm{B}_{2}$, 6-keto-PGF $\mathrm{F}_{\mathrm{la}}$ in patients with primary hyperlipidaemia.

Methods 120 patients with primary hyperlipidaemia were enrolled in this study, 82 males and 38 females, age $36-74$ years old, average ages (55 \pm 9 ) years old. 12 weeks after taking Xuezhikang, the clinical effect and the effect on the level of plasma endothelins, thromboxane $\mathrm{B}_{2}, 6$-Keto- $\mathrm{PGF}_{\text {la }}$ were compared before and after the treatment, and the relation between blood lipids and ratio of plasma endothelins, thromboxane $\mathrm{B}_{2}$ to 6-Keto-PGF $\mathrm{Pa}_{\mathrm{la}}$ were analysed.

Results 12 weeks after treatment, the level of TG, cholesterol (TC), low density lipoprotein cholesterol (LDL-C) and $\mathrm{apoB}_{100}$ decreased sharply $(p<0.05-0.001)$; the level of serum high density lipoprotein cholesterol (HDL-C) elevated $(p<0.05)$; ratio of plasma endothelins decreased sharply $(p<0.001)$; rate value of thromboxane $B_{2}$ to 6 Keto-PGF $\mathrm{F}_{\mathrm{la}}$ before treatment was higher than health people but lower after treatment $(p<0.01)$. There were positive correlations between the decreased TC. TG, LDL-C and decreased ET-1, the ratio $\mathrm{TXB}_{2} / 6$-keto-PGF ${ }_{\text {la }}(\mathrm{r}=0.832-0.963, \mathrm{p}<0.01-0.001)$. The same positive correlation was found between the decreased ET-1 and the ratio of $\mathrm{TXB}_{2} / 6$-keto-PGF $1 \mathrm{a}(\mathrm{r}=0.987, \mathrm{p}<0.001)$. 